\title{
A Potent Leukocyte Transmigration Blocker: GT-73 Showed a Protective Effect against LPS-Induced ARDS in Mice
}

\author{
Eliav Blum ${ }^{1}{ }^{\mathbb{D}}$, Raanan Margalit ${ }^{2}$, Laura Levy ${ }^{1}$, Tamar Getter ${ }^{1}$, Ron Lahav ${ }^{3}$, Sofia Zilber ${ }^{4}$, Paul Bradfield ${ }^{5}$, \\ Beat A. Imhof ${ }^{6} \mathbb{D}$, Evgenia Alpert ${ }^{3, *}$ and Arie Gruzman ${ }^{1, *}$ \\ 1 Department of Chemistry, Faculty of Exact Sciences, Campus Ramat-Gan, Bar-Ilan University, \\ Ramat-Gan 5290002, Israel; eliavblum@gmail.com (E.B.); levylaura55@hotmail.com (L.L.); \\ tamargetter25@gmail.com (T.G.) \\ 2 Science in Action, 3 Pinchas Sapir Street, Weizmann Science Park, Ness-Ziona 7403650, Israel; \\ raanan.margalit@gmail.com \\ 3 AltA-ZuZ Therapeutics, 3 Pinchas Sapir Street, Weizmann Science Park, Ness-Ziona 7403650, Israel; \\ lahav.r@gmail.com \\ 4 Department of Pathology, Shaare Zedek Medical Center, 12 Shmuel Bait Street, Jerusalem 9103102, Israel; \\ sofiazilber@szmc.org.il \\ 5 MesenFlow Technologies, Chemin des Aulx, 14, CH-1228 Geneva, Switzerland; \\ paul.bradfield@mesenflow.com \\ 6 Department of Pathology and Immunology, University of Geneva, Rue Michel-Servet, CH-1211 Geneva, \\ Switzerland; beat.imhof@unige.ch \\ * Correspondence: genia.alp@gmail.com (E.A.); gruzmaa@biu.ac.il (A.G.)
}

check for updates

Citation: Blum, E.; Margalit, R.; Levy, L.; Getter, T.; Lahav, R.; Zilber, S.; Bradfield, P.; Imhof, B.A.; Alpert, E.; Gruzman, A. A Potent Leukocyte Transmigration Blocker: GT-73 Showed a Protective Effect against LPS-Induced ARDS in Mice. Molecules 2021, 26, 4583. https:// doi.org/10.3390/molecules26154583

Academic Editor: Kenneth H. Pearce

Received: 13 June 2021

Accepted: 22 July 2021

Published: 29 July 2021

Publisher's Note: MDPI stays neutral with regard to jurisdictional claims in published maps and institutional affiliations.

Copyright: (c) 2021 by the authors. Licensee MDPI, Basel, Switzerland. This article is an open access article distributed under the terms and conditions of the Creative Commons Attribution (CC BY) license (https:// creativecommons.org/licenses/by/ $4.0 /)$.

\begin{abstract}
We recently developed a molecule (GT-73) that blocked leukocyte transendothelial migration from blood to the peripheral tissues, supposedly by affecting the platelet endothelial cell adhesion molecule (PECAM-1) function. GT-73 was tested in an LPS-induced acute respiratory distress syndrome (ARDS) mouse model. The rationale for this is based on the finding that the mortality of COVID-19 patients is partly caused by ARDS induced by a massive migration of leukocytes to the lungs. In addition, the role of tert-butyl and methyl ester moieties in the biological effect of GT-73 was investigated. A human leukocyte, transendothelial migration assay was applied to validate the blocking effect of GT-73 derivatives. Finally, a mouse model of LPS-induced ARDS was used to evaluate the histological and biochemical effects of GT-73. The obtained results showed that GT-73 has a unique structure that is responsible for its biological activity; two of its chemical moieties (tert-butyl and a methyl ester) are critical for this effect. GT-73 is a prodrug, and its lipophilic tail covalently binds to PECAM-1 via Lys536. GT-73 significantly decreased the number of infiltrating leukocytes in the lungs and reduced the inflammation level. Finally, GT-73 reduced the levels of IL-1 $\beta$, IL-6, and MCP-1 in bronchoalveolar lavage fluid (BALF). In summary, we concluded that GT-73, a blocker of white blood cell transendothelial migration, has a favorable profile as a drug candidate for the treatment of ARDS in COVID-19 patients.
\end{abstract}

Keywords: ARDS; leukocyte transmigration; cytokine storm; PECAM-1; covalent inhibitor

\section{Introduction}

COVID-19 is a new viral disease affecting many human organs, predominantly targeting the respiratory system and the lungs. All of us are currently trying to survive this deadly global pandemic that seriously challenges and limits public health systems around the world. In about $20 \%$ of patients, severe symptoms occur after a mean incubation period of five days. Furthermore, $5 \%$ of patients need intensive care therapy, including ventilation support [1-3].

The main way of obtaining COVID-19 treatment is based on established recommendations for treating patients with acute lung injury (ARDS) and "cytokine storm". The IL-6 level, in particular, should be reduced dramatically to block the severe results of 
the "cytokine storm" [4]. Lung ventilation (protective and functional), anti-inflammatory therapy, diuretics, and an adequate management of organ failures constitute the milestones of therapy. In case of significant lung failure, venovenous extracorporeal membrane oxygenation may also be used [5].

ARDS is a major reason for mortality in COVID-19 patients [6,7]. In advanced and especially in the terminal stages of the disease, leukocytes accumulate in the lungs and occupy mainly the alveolar space, including the interstitium due to inflammation induced by viral infection. This first leads to a "cytokine storm", followed by a devastating destruction of the alveolar tissues [8]. During this stage, the immune system mainly attacks the host tissue; the adaptive immune cells are not yet effective in fighting the virus by antibodies or the specific killing of infected host cells [9]. Thus, blocking this process may successfully treat and reverse the effects of ARDS in COVID-19 patients, similar to the results obtained with our in vivo animal model.

We recently developed a potent inhibitor of leukocyte transmigration from the blood to the tissues (GT-73). This compound was also able to increase the reverse transmigration of monocytes [10]. Compound GT-73, based on the scaffolding of barbituric acid, has already shown highly promising results as a drug candidate for the treatment of other inflammatory and autoimmune disorders (e.g., Crohn's disease, multiple sclerosis, fatty liver disease, inflammatory bowel disease, and rheumatoid arthritis). This was achieved without any toxic effects on human endothelial cells in vitro. In addition, GT-73 was not toxic in vivo, even when it was used at suprapharmacological doses.

Despite these promising preliminary results, GT-73's suggested mechanism of action (blocking PECAM-1 activity) has not been elucidated. We previously reported that according to in silico calculations, GT-73 might affect PECAM-1 activity. PECAM-1 is one of the vascular adhesion molecules that play an important role in leukocyte transendothelial migration during inflammation and cancer [11-13]. PECAM-1 not only resides at endothelial junctions, but it is also a constituent of a recycling compartment of endothelial cells, termed the lateral border recycling compartment (LBRC), which most likely provides PECAM-1 molecules to junctions during leukocyte transmigration [14,15]. It was shown that high levels of PECAM-1 lead to increased transendothelial migration of immune cells from blood to organs [16-19].

Here, we describe the substantial therapeutic effect of GT-73 in LPS-induced ARDS in mice. GT-73 was administered intranasally and had a significant positive effect on the severity of the "cytokine storm" and on lung morphology. We also found that PECAM-1 is indeed a primary target of GT-73 activity.

Finally, two critical residues for the biological effect of moieties on GT-73 were identified: tert-butyl and methyl ester. The absence of these functional groups in the structure of GT-73 completely abolishes its biological activity.

\section{Results}

\subsection{Structure-Activity Relationships (SARs) and a Possible Mechanism Underlying GT-73 Action}

In our previous publication, we reported the screening results (the blocking of leukocyte transendothelial migration) of a series of lipophilic compounds [10]. We carefully studied the structural differences between the nonblocking (GT-77) and the completely blocking (GT-73) compounds [10]. There are two differences between these molecules: (1) the addition of the tert-butyl group on the central aromatic ring and (2) the replacement of carboxylic acid with an ester moiety by the addition of a methoxy group. Thus, the importance of both of these chemical moieties in enhancing the biological activity was investigated.

Two novel compounds (EB-237 and EB-269) were synthesized to determine the importance of the two structural moieties of GT-73 (methyl ester and tert-butyl) in enhancing the biological effect. The synthesis was initiated from the commercial materials 4(chloromethyl) benzoic acid and 4-(bromomethyl) benzoic acid, respectively, using Knoevenagel condensation [20] to obtain EB-237 and EB-269, respectively, as described in 
Supplementary Schemes S1 and S2. Both compounds were tested for their ability to bridge the gap between GT-77 and GT-73 [10]. Therefore, we added to the basic scaffold (GT-77) only one of the supposed functional groups at that time to obtain compounds EB-237 and EB-269. Next, the effect of both compounds GT-73 and its fluorinated version termed EB251 (Chart 1 and Supplementary Scheme S2) on the transendothelial migration of primary, human monocytes was investigated under flow conditions using inflammatory, TNF- $\alpha$ activated HUVECs. GT-73 and EB-251 exhibited a significant time-dependent inhibition of monocyte transmigration from two independent donors (Figure 1). In contrast, the novel modified compounds EB-237 and EB-269 did not block this process (Figure 1).<smiles>O=C1NC(=O)C(=Cc2ccccc2OCc2ccc(C(=O)O)cc2)C(=O)N1</smiles>

Compound GT-77<smiles>COC(=O)c1ccc(COc2c(C=C3C(=O)NC(=O)NC3=O)cccc2C(C)(C)C)cc1</smiles>

Compound GT-73<smiles>COC(=O)c1ccc(COc2ccccc2C=C2C(=O)NC(=O)NC2=O)cc1</smiles>

EB-237 Compound 3<smiles>COC(=O)c1ccc(COc2c(C=C3C(=O)NC(=O)NC3=O)cccc2C(C)(C)C)cc1F</smiles>

EB-251 Compound 6<smiles>CC(C)(C)OC(=O)OCc1ccc(COc2c(C=C3C(=O)NC(=O)NC3=O)cccc2C(C)(C)C)cc1</smiles>

Compound 8

Chart 1. Structures of the synthesized compounds.

Next, GT-73's mechanism of action was investigated. According to in silico calculations, the protein affected by GT-73 during monocyte transmigration may be PECAM-1 [10]. Furthermore, we hypothesized that GT-73 could act as a prodrug that binds covalently to PECAM-1. Therefore, the nonstable bond between barbituric acid and the lipophilic moiety might be cleaved by a nucleophilic attack of the primary amine of a lysine located in PECAM-1. Such an attack will result in the formation of a covalent imine bond between the amino acid of the protein and the lipophilic moiety of GT-73 (Supplementary Chart S1). To test this hypothesis, we used mass spectroscopy. To this end, recombinant human PECAM-1 was incubated with GT-73. However, under these conditions, the imine bond would be cleaved, and identifying a covalently attached fragment to PECAM-1 would have been impossible. To avoid this scenario, we added sodium borohydride to the reaction mix before trypsinization, which induces the reduction of the imine bond to a secondary amine, as shown in Supplementary Chart S1. Secondary amines are stable under acidic $\mathrm{pH}$ conditions when PECAM-1 is trypsinized as a part of the sample preparation for the mass spec analysis. When inspecting the protein sequence of PECAM-1, we could identify a lipophilic tail of GT-73 as a covalent adduct to Lys ${ }^{536}$, as shown in Supplementary Figure S1. 
A
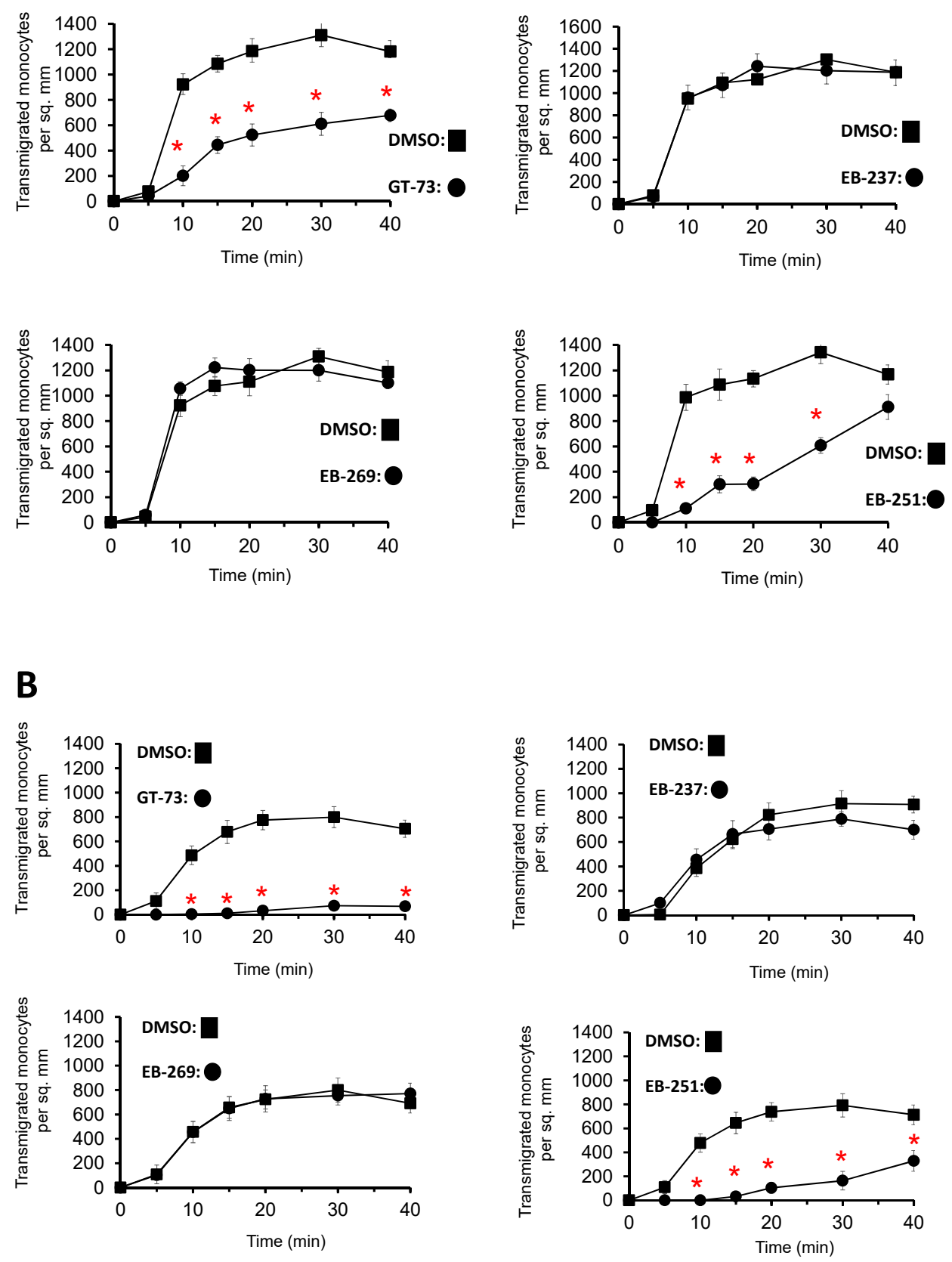

Figure 1. Monocyte transmigration (absolute number) on the surface of the activated HUVECs under flow. Primary human monocytes were cocultured on TNF- $\alpha$-activated HUVECs. Test compounds at $30 \mu \mathrm{M}$ or DMSO $(0.1 \%)$ were added to cells for $45 \mathrm{~min}$. Cocultures were kept under a flow of 0.05 Pa. Mean $\pm \mathrm{SE},{ }^{*} p \leq 0.05$. (A) First donor. (B) Second donor.

\subsection{In Vivo Investigation}

Knowing that GT-73 can covalently bind to PECAM- 1 and that it most likely affects its function, we tested GT-73 in a pathology model that leads to severe/terminal stages of COVID-19: ARDS. During the development of ARDS, leukocytes migrate into the lungs, which is accompanied by a "cytokine storm". We used BALB/c female mice and induced ARDS with intratracheal instillation of LPS. Treatment with GT-73 and vehicle, also administered intratracheally, began $3 \mathrm{~h}$ after LPS application. The experiment was then terminated at $72 \mathrm{~h}$. The effect of GT-73 on the number of infiltrating leukocytes in the lungs and the cytokine levels in bronchoalveolar lavage fluid (BALF) were analyzed, as 
shown in Figure 2A,B, respectively. GT-73 significantly reduced the number of infiltrating leukocytes and significantly reduced the levels of IL-1 $\beta$, IL-6, and MCP-1 in BALF.
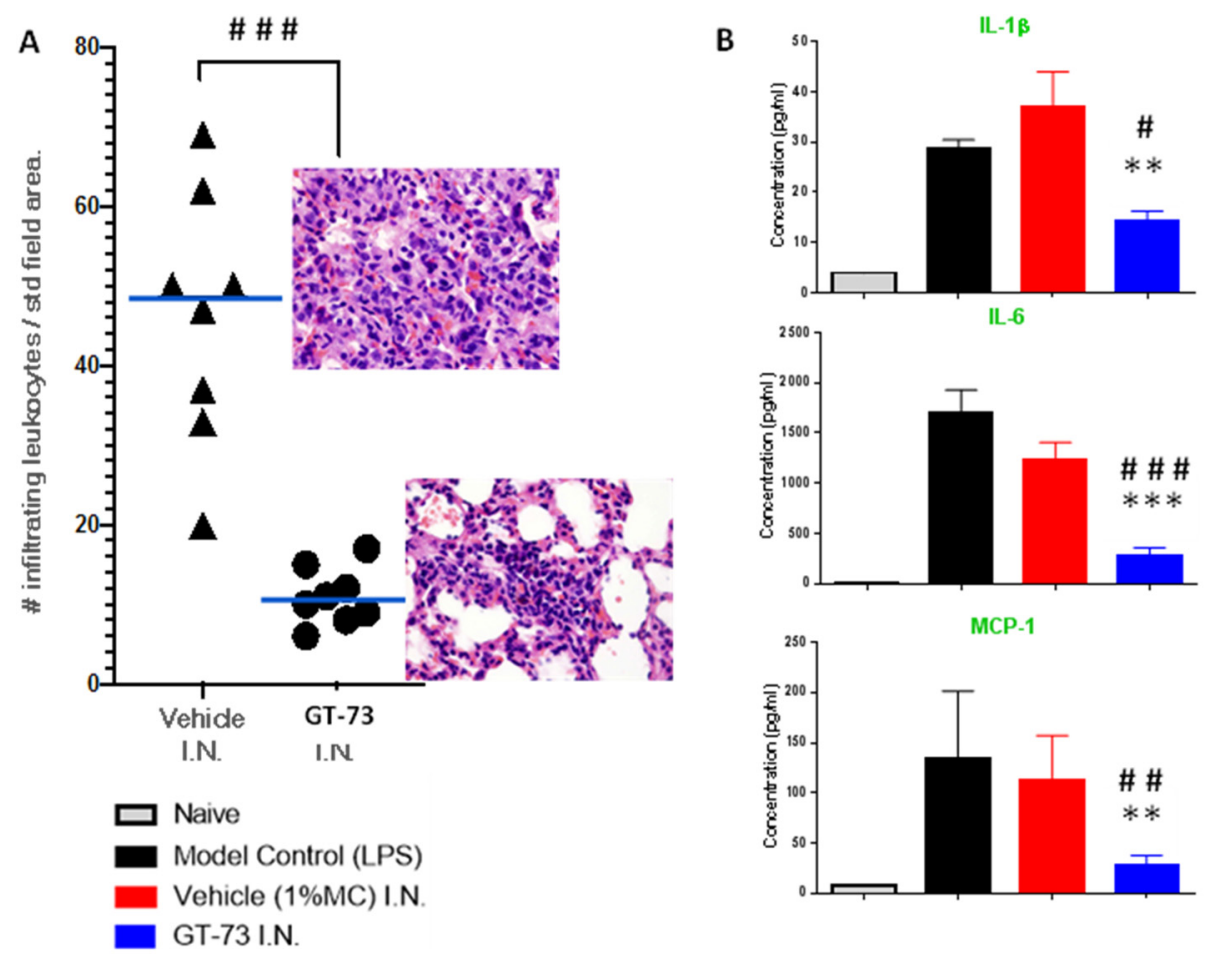

Figure 2. In vivo effect of GT-73. (A) Effect of GT-73 on the number of leukocytes in the lungs. Female BALB/c mice were anesthetized and orally intubated with a catheter and challenged with intratracheal instillation LPS $(800 \mu \mathrm{g})$ in PBS. Mice were euthanized at $3 \mathrm{~d}$ after LPS challenge to collect tissues for analysis. All animals from the treatment group were administered GT-73 (5 mg/ kg in $1 \%$ methyl cellulose) for $3 \mathrm{~h}$ after LPS application by the intranasal route 3 times a day. Lungs were collected in $10 \%$ buffered formalin, embedded in paraffin, and cut into sections. Sections were stained with hematoxylin/eosin, and images were taken with an Olympus BX53 microscope. (B) Samples of BALF were centrifuged. The supernatant was flash-frozen, and the cytokine levels were measured. $n=7, \mathrm{MEAN} \pm \mathrm{SD}$, significant difference between treated by GT-73 and vehicle groups, ${ }^{*} p \leq 0.004$, \#\# $p \leq 0.003$, and ${ }^{\# \#} p \leq 0.0001$. Significant difference between treated by GT-73 and model control groups $^{* *} p \leq 0.003$, and ${ }^{* * *} p \leq 0.0001$.

To evaluate the potential role of GT-73 on the histopathological changes in the lungs, we produced paraffin-embedded tissue sections and stained them with hematoxylin/eosin. The inflammatory infiltrates, interalveolar septal thickening, and interstitial edema were evaluated (Figure 3A-D). Administration of GT-73 efficiently reduced the airspace inflammation. Furthermore, the severity of lung injury was also assessed using a semiquantitative histopathology score system, which evaluates lung injury according to four categories: alveolar septae, alveolar hemorrhage, intra-alveolar fibrin, and intra-alveolar infiltrates.

Vehicle and LPS control groups exhibited a prominent neutrophilic infiltrate in the alveolar space and the interstitial septa, but the extent was variable both within and across lobes and in neutrophilic debris deposits, numerous lymphocytes, and macrophages (Figure 3E-K). The ARDS induction affected the air-blood barrier, leading to a progressive breakdown of the capillary walls and the epithelial integrity, which permitted leakage of protein-rich edema fluid (interstitial fluid and plasma proteins) into the alveoli. This proteinaceous fluid presented itself as a lightly eosinophilic material that ranges from homogeneous to fibrillar (i.e., fibrin strands) in appearance in the H\&E-stained sections and is present to a variable extent in all specimens. Reactive pneumocytes showed desquamation into alveoli. All specimens collected from the GT-73-treated group showed a diffuse distri- 
bution of ill-defined low-density inflammatory lesions, with mild to moderate neutrophilic infiltrate into the alveolar space and interstitial septae, but without neutrophilic debris deposits or lymphocytes; however, there was a higher number of foaming macrophages (indicating the start of the repair phase). In contrast, specimens collected from nontreated mice exhibited huge amounts of neutrophilic debris, high numbers of lymphocytes, and almost no macrophages. In addition, in the treated mice we detected minimal numbers of focal reactive pneumocytes with desquamation into alveoli. Overall, we found that treatment with GT-73 significantly reduced the number of these lung injury scores (Figure 3K).

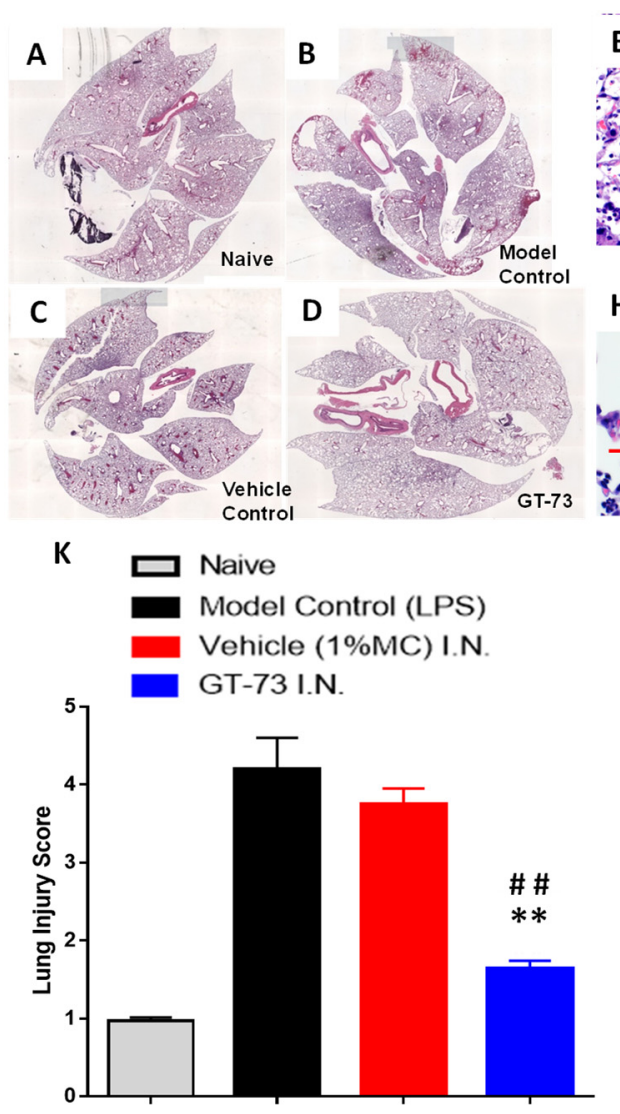

Figure 3. Histological evaluation of the lung inflammation level. Slides were prepared and processed as described in Figure 2. Images of whole-mount lungs were taken at low magnification $(\times 20)$ : (A) naive mice; (B) untreated model control (LPS); (C) vehicle-treated lungs; (D) GT-73-treated lungs. Images were taken at high magnification $(\times 400)$. Upper panel, vehicle-treated lungs: (E) fibrin deposits; (F) septal and alveolar neutrophils infiltration. (G) neutrophilic debris deposits. Lower panel, GT-73-treated lungs: (H) alveolar neutrophil infiltration and foaming macrophages; (I) septal neutrophil infiltration. (J) normal lungs (naïve); (K) Lung injury histological score, $n=7$, mean $\pm \mathrm{SD}$, significant difference between treated by GT-73 and the vehicle groups, ${ }^{\# \#} p \leq 0.003$. Significant difference between treated by GT-73 and the model control groups $^{* *} p \leq 0.003$.

\section{Discussion}

The presented results substantially enhance our knowledge about GT-73's mechanism of action and elucidate its function in a COVID-19-relevant inflammatory pathology. It was important to confirm that the chemical structure of GT-73 has two functional moieties: tert-butyl and methyl ester, both of which are essential for maintaining the biological activity. Only the replacement of hydrogen by a fluorine atom in the second aromatic ring left the activity intact. The absence of both functional groups in the GT-73 structure led to the complete loss of biological activity. These results might indicate that these two lipophilic groups are required for the binding between GT-73 and the targeted protein. This hypothesis will be investigated in future work. 
We determined, as an adduct to the PECAM-1 sequence, only part of GT-73. Therefore, GT-73 is a classical prodrug and disintegrates after the nucleophilic attack of lys536 in PECAM-1 into two molecules: one is a leaving group (barbituric acid), and the other is the lipophilic alkyl tail, which covalently binds to the lysine by forming an imine bond. Imine bonds can undergo hydrolysis; thus, the covalent inhibition of PECAM-1 is reversible. This may be of importance for therapy and it explains the total absence of toxicity, which was previously reported by our laboratories [10]. The covalent addition of a lipophilic moiety to the extracellular domain of PECAM-1 (lys536 is located extracellularly) may be critical for its function, and it should seriously interrupt the function of PECAM-1, an important adhesion molecule in inflammation-driven leukocyte migration. It might be the basis for the therapeutic effect of GT-73, which we observed in the treatment of ARDS and in other in vivo autoimmune disease models [10].

ARDS contributes to COVID-19-induced patient death. Older age and additional comorbidities increase the rate of mortality from ARDS [21]. ARDS in patients begins with an augmented permeability of alveolar capillaries for proteins such as albumin, fibrinogen, proinflammatory cytokines, and coagulation factors [22]. The next step is the massive transendothelial migration of inflammatory leukocytes, including neutrophils to the lungs. Such a massive accumulation of white blood cells leads to a severe local inflammatory response, a "cytokine storm", or fibrin deposition; all these factors induce the progression of respiratory dysfunction and heart failure [23]. Recently, suppression of the accumulation of white blood cells in the lungs of COVID-19 patients by dexamethasone (a glucocorticoid that was recently shown to be the first life-saving drug in this disease) was recommended as a mandatory part of the treatment because no other therapies have shown a positive effect during the late or terminal stages of the disease [24]. Here and in our previous publication, we showed that GT-73 inhibits the transmigration of white blood cells [10]. This led to a significantly improved lung morphology after induction of ARDS by LPS. In treated mice, the lungs were cleared from neutrophils and cell debris. GT-73 caused a significant reduction in the number of infiltrated leukocytes but increased the number of macrophages. The latter indicates that the recovery stage in the local inflammatory response had already started. It is known that macrophages play different roles in inflammation, depending on their differentiation stages. In the first (the acute) phase, alveolar macrophages resemble an activated, inflammatory phenotype (M1 in humans); they secrete proinflammatory mediators and factors. In the later phase, the M1 phenotype changes to a rather M2 antiinflammatory phenotype, and this change allows them to eliminate apoptotic cells, cell debris, and induces the replacement of the dead alveoli by connective tissues $[25,26]$.

Cytokines are deeply involved in the development of inflammation [27,28]. During the onset of an antiviral response, cytokines such as interferons play an important role in the induction of an anti-inflammatory response, but at later stages, other types of cytokines in critical-condition patients can lead to severe tissue damage, as they induce the release of toxic reagents by leukocytes such as cytotoxic reactive oxygen species [29]. Therefore, reducing the extent of the "cytokine storm" is a mandatory step in the treatment of COVID-19 patients. It was recently reported that in patients with severe COVID-19 the levels of IL-2, IL-7, IL-10, GSCF, IP10, MCP-1, MIP1A, and TNF- $\alpha$ are dramatically elevated [30]. In addition, the proinflammatory cytokines IL- 6 and IL- $1 \beta$ are also elevated in ARDS patients. This leads to a higher expression of cell adhesion molecules by vascular endothelium and VEGF secretion. The latter augments the vascular permeability of the lung endothelium, allowing quick viral dissemination; the former induces a massive transmigration of neutrophils and inflammatory monocytes from the blood to the lungs. IL6 and IL-1 $\beta$ also stimulate the proliferation and differentiation of hematopoietic progenitors in bone marrow. As immature granulocytes, they then can go directly to the struggling lungs and further increase local inflammation, which can lead to ARDS. This chain of events in the lung-systemic loop is responsible for the cytokine storm syndrome [31]. GT-73 exhibited significant inhibitory effects on the cytokine level of three cytokines: IL-1 $\beta$, IL-6, 
and MCP-1 in the lungs. However, this effect might turn into an important advantage in the fight against the cytokine storm associated with ARDS.

\section{Materials and Methods}

All chemical reagents, solvents, and acids were purchased from Acros Organic (Fair Lawn, NJ, USA), Alfa Aesar (Ward Hill, MA, USA), Bio-Lab Ltd. (Jerusalem, Israel), and Merck (Rehovot, Israel), and all were used as received. LPS from Salmonella enteritidis and methyl cellulose were from Merk (Rehovot, Israel). Anhydrous tetrahydrofuran (THF) was obtained by distillation from a boiled blue mix containing sodium $(1 \% w / v)$ and benzophenone $(0.2 \% w / v)$. Column chromatography was performed on silica gel 60 (230-400 mesh; Merck, Darmstadt, Germany). Analytical and preparative high-performance liquid chromatography (HPLC) (Young Lin Instruments, Anyang, South Korea) were performed on LUNA C18 preparative $(10 \mu \mathrm{m}, 100 \times 30 \mathrm{~mm})$ or analytical $(5 \mu \mathrm{m}, 250 \times 4.6 \mathrm{~mm})$ columns, both from Phenomenex, Inc. (Torrance, CA, USA). HPLC purification was carried out with an increasing linear gradient of $\mathrm{CH}_{3} \mathrm{CN}$ in $\mathrm{H}_{2} \mathrm{O}$. The purity of the synthesized compounds was confirmed by HPLC analysis and elemental analysis. Analytical thin-layer chromatography (TLC) was carried out on pre-coated silica gel 60 F254 (Merck, Darmstadt, Germany) sheets using UV absorption and iodine physical adsorption for visualization. Mass spectra were recorded on a Finnigan Model 400 instrument using a QToF microspectrometer (Micromass, Milford, MA, USA), using electrospray ionization (ESI) in the positive ion mode. Data were processed using mass L-ynX ver. 4.1 calculation and deconvolution software (Waters Corp., Milford, MA, USA). High-resolution mass spectra (HRMS) were obtained using an LTQ Orbitrap XL (Thermo Scientific, Waltham, MA, USA). Melting points were measured with a Fisher-Johns melting point apparatus (Waltham, MA, USA). The ${ }^{1} \mathrm{H}$ NMR and ${ }^{13} \mathrm{C}$ NMR were recorded at room temperature on a Bruker AVANCE NMR spectrometer (Vernon Hills, IL, USA) operating at 300, 400, 600, and $700 \mathrm{MHz}$ and were in accordance with the assigned structures. Chemical shift values were reported relative to tetramethylsilane (TMS), which was used as an internal standard. Chemical shifts were expressed in $\delta(\mathrm{ppm})$ and coupling constants $(J)$ were in hertz. The splitting pattern abbreviations are as follows: $s$, singlet; $d$, doublet; $t$, triplet; $q$, quartet; quint, quintet; $\mathrm{m}$, unresolved multiplet due to the field strength of the instrument; dd, doublet of doublets.

\subsection{In Vivo Study}

\subsubsection{Mice}

Female BALB/c mice, 6-8 weeks old, were purchased from Envigo (Jerusalem, Israel).

\subsubsection{Murine Model of LPS Induced ARDS}

The murine model of LPS-induced ARDS was established as reported ${ }^{19}$. Briefly, female BALB/c mice ( $n=8$ per group) were anesthetized and orally intubated with a sterile plastic catheter and challenged with an intratracheal instillation of $800 \mu \mathrm{g}$ of LPS (E. coli 055:B5; Merck, Rehovot, Israel) dissolved in $50 \mu \mathrm{L}$ of normal PBS. Naïve mice (without LPS instillation) were injected with the same volume of pyrogen-free PBS to serve as controls. Mice were euthanized $3 \mathrm{~d}$ after LPS challenge to collect tissues for analysis.

All animals from the treatment group were administered $20 \mu \mathrm{L}$ of GT-73 at a $10 \mathrm{mg} / \mathrm{mL}$ concentration in $1 \%$ methyl cellulose by intranasal route 3 times a day $3 \mathrm{~h}$ after LPS application. All animals from the vehicle control group were administered $20 \mu \mathrm{L}$ of $1 \%$ methyl cellulose by intranasal route 3 times a day $3 \mathrm{~h}$ after LPS application. Finally, animals from the LPS control group were used as an experimental nontreated model control. Naïve animals were used as a baseline control. The drug candidate was administered at a constant volume dosage for $72 \mathrm{~h}$ following LPS administration. 


\subsubsection{Tissue Collection}

Samples of Bronchial Alveolar Lavage fluid (BALF) were collected from each mouse from all groups; they were centrifuged for supernatant and cell pellet separation. The supernatant was snap-frozen to determine the cytokine levels, and cells were used for FACS white cell differentiation. Lungs were collected and filled with $10 \%$ buffered formalin, embedded in paraffin, and cut into $4 \mu \mathrm{m}$ thick sections. Sections were stained with hematoxylin/eosin (H\&E), and images were taken with an Olympus BX53 microscope $(400 \times)$. For the lung injury score, images were evaluated by an investigator who was blinded to the identity of the slides. In brief, the extent of the pathological lesions was graded from 0 to 3 (see Table 1). The score for each animal was calculated by dividing the total score by the number of sections observed.

Table 1. Histological score.

\begin{tabular}{ccccc}
\hline Score & Alveolar Septae & Alveolar Hemorrhage & Intra-Alveolar Fibrin & $\begin{array}{c}\text { Intra-Alveola } \\
\text { Infiltrations per Field }\end{array}$ \\
\hline 0 & All are thin and delicate & No hemorrhage & No intra-alveolar fibrin & $\begin{array}{c}\text { Less than } 5 \\
\text { intra-alveolar cells }\end{array}$ \\
\hline 1 & $\begin{array}{c}\text { Congested alveolar septae in } \\
\text { less than } 1 / 3 \text { of the field }\end{array}$ & $\begin{array}{c}\text { Erythrocytes per alveolus in } \\
1 \text { to } 5 \text { alveoli }\end{array}$ & $\begin{array}{c}\text { Fibrin strands in less } \\
\text { than } 1 / 3 \text { of the field }\end{array}$ & intra-alveolar cells \\
\hline 2 & $\begin{array}{c}\text { Congested alveolar septae in } \\
1 / 3 \text { to } 2 / 3 \text { of the field }\end{array}$ & $\begin{array}{c}\text { At least } 5 \text { erythrocytes per } \\
\text { alveolus in } 5 \text { to } 10 \text { alveoli }\end{array}$ & $\begin{array}{c}\text { Fibrin strands in } 1 / 3 \text { to } \\
2 / 3 \text { of the field }\end{array}$ & intra-alveolar cells \\
\hline 3 & $\begin{array}{c}\text { Congested alveolar septae in } \\
\text { more than } 2 / 3 \text { of the field }\end{array}$ & $\begin{array}{c}\text { At least } 5 \text { erythrocytes per } \\
\text { alveolus in more than } \\
10 \text { alveoli }\end{array}$ & $\begin{array}{c}\text { Fibrin strands in more } \\
\text { than } 2 / 3 \text { of the field }\end{array}$ & $\begin{array}{c}\text { More than 20 } \\
\text { intra-alveolar cells }\end{array}$ \\
\hline
\end{tabular}

\subsubsection{Histological Evaluation of the Lung Inflammation Level}

The histological analysis was conducted based on the criteria listed in Table 1 [32].

\subsection{In Vitro Human Monocyte Trafficking Assay}

Leukocyte trafficking assays were conducted as described previously. [10] Briefly, human umbilical vein endothelial cells (HUVECs) were isolated by collagenase treatment of umbilical veins and maintained (passages 2-4) in Medium 199 containing 10\% FCS, $15 \mu \mathrm{g} / \mathrm{mL}$ endothelial cell growth supplement (Upstate Biotechnology, Lake Placid, NY, USA), $100 \mu \mathrm{g} / \mathrm{mL}$ heparin, and $50 \mu \mathrm{M}$ hydrocortisone. For the flow assays, HUVECs were cultured after a maximum of 3-5 passages in chamber slides (m-slide VI 0.1 ibiTreat, IBIDI), previously coated with $0.2 \%$ gelatin (Merck, Darmstadt, Germany) and $1 \mathrm{mg} / \mathrm{mL}$ Collagen $\mathrm{G}$ (Biochrom AG, Berlin, Germany) for 2-3 days and then treated for $24 \mathrm{~h}$ with acute or chronic inflammation protocols using tumor necrosis factor $\alpha$ (TNF- $\alpha$ ) at $1000 \mathrm{U} / \mathrm{mL}$ alone or in combination with interferon $\gamma(\operatorname{IFN} \gamma)$, at $500 \mathrm{U} / \mathrm{mL}$ for chronic inflammatory protocols. [10] The compounds to be tested and the leukocytes were added at $30 \mu \mathrm{M}$. Monocytes were purified from EDTA blood collected from healthy donors using Ficoll gradients and negative selection kits (Miltenyi Biotec, Bergisch Gladbach, Germany) for those types of cells. The slides containing the cultured HUVEC monolayers were then mounted to a flow system set at $37^{\circ} \mathrm{C}$, and flow was generated over the HUVEC monolayer by perfusing a wash medium or a medium containing the leukocyte suspension using a calibrated pump. The flow rate, expressed as a measurement of shear stress, represented small venules/capillaries (0.05 Pascal (Pa)). The first stage of the assay was a washing procedure, whereby the wash buffer was perfused over the HUVECs for $10 \mathrm{~min}$. Wash buffer was then perfused over the HUVECs for 10 min to remove unbound chemokines, before purified leukocytes were perfused over the HUVECs for $5 \mathrm{~min}$, followed by $45 \mathrm{~min}$ with wash buffer.

Adhesion events were recorded as the total number of cells per unit field $\left(\mathrm{mm}^{2}\right)$. Transmigration events are presented as a percentage of the total leukocytes captured 
from flow per unit field. All experiments were carried out using triplicate fields and are presented as a mean value with +standard error measurements (+SEM). Statistical analyses assume a parametric distribution and were conducted using Student's $t$-test.

\subsection{Cytokine Level Determination}

The levels of IL-1 $\beta$, IL- 6 , and MCP- 1 were determined using commercially available ELISA analytical kits (Merck, Rehovot, Israel). The assays were conducted according to the protocols supplied by the manufacturer.

\subsection{Synthetic Chemistry}

The synthesis and the analytical data for the obtained molecules are presented in the Supplementary Materials.

\subsection{Mass Spectroscopy}

Human recombinant PECAM-1(Seale, WA, USA) $(25 \mu \mathrm{g})$ was dissolved in PBS buffer, (Merck, Rehovot, Israel) $(30 \mu \mathrm{L})$. GT-73 was added to the solution in $0.5 \mu \mathrm{L}$ of DMSO to reach a final concentration of $30 \mu \mathrm{M}$. The reaction was incubated under gentle agitation for 40 min at $37^{\circ} \mathrm{C}$. Then, $\mathrm{NaBH}_{4}$ in PBS $(20 \mu \mathrm{L})$ was added to obtain a final concentration of $100 \mathrm{mM}$, and the reaction mix was kept for an additional incubation time $\left(1 \mathrm{~h}\right.$, at $\left.37^{\circ} \mathrm{C}\right)$. The sample was digested by trypsin, analyzed by LC MS/MS on Q-Exactive (Thermo Fisher, Waltham, MA, USA), and identified by Discoverer software against Uniprot unspecific and specific databases. All the identified peptides were filtered with high confidence, top rank, and mass accuracy. High confidence peptides passed the 1\% FDR threshold $\left({ }^{*} \mathrm{FDR}=\right.$ false discovery rate; it is the estimated fraction of false positives in a list of peptides). Semiquantitation was performed by calculating the peak area of each peptide.

\section{Conclusions}

We recently developed an inhibitor of leukocyte transmigration from the blood to the peripheral tissues (GT-73). Here, we showed that GT-73 has a unique structure, which is responsible for its biological activity, and two of its chemical moieties (tert-butyl and a methyl ester) are critical for this effect. In addition, we showed that GT-73 is a prodrug, and its lipophilic tail covalently binds to PECAM- 1 via $\mathrm{Lys}^{536}$, forming an imine bond.

In an LPS-induced ARDS mouse model, GT-73 was given intranasally during the induction phase of ARDS ( $3 \mathrm{~h}$ after the intranasal administration of LPS), with encouraging results. Specifically, all specimens collected from treated animals by the compound group showed a diffuse distribution of ill-defined low-density inflammatory lesions, with mild to moderate neutrophilic infiltrate into the alveolar space and interstitial septa, but without neutrophilic debris deposits or lymphocytic infiltrates, as well as a higher number of foaming macrophages (an indication of a repair phase). In contrast, specimens collected from untreated mice exhibited huge amounts of neutrophilic debris and lymphocytic infiltrates and almost no macrophages. In addition, the treated mice exhibited only minimal amounts of reactive pneumocytes with focal desquamation into the alveolar space, compared with untreated mice, where the number of reactive pneumocytes and desquamation was much higher. Finally, GT-73 significantly reduced the number of infiltrating leukocytes and reduced the level of a "cytokine storm", manifested by lower levels of IL-1 $\beta$, IL-6, and $\mathrm{MCP}-1$ in bronchial lavage fluid.

\section{Patent}

The GT-73 structure and its use as a potential therapeutic agent are protected by a patent: Gruzman et al. (2019). "Novel barbituric acid-based leucocyte transmigration inhibitors as drug candidates for treating inflammatory diseases, autoimmune diseases and cancer". WO2019043706A1. 
Supplementary Materials: The following are available online. The synthetic chemistry part, PECAM1 covalent modification by GT-73, analytical chemistry data, and the purity level of the synthesized compounds are available online, Scheme S1: Synthetic pathway of ester derivative 3 (EB-237), Scheme S2: Synthetic pathway of fluorinated derivative 6 (EB-251), Scheme S3: Synthetic pathway of carboxylic acid derivative 8 (EB-269), Chart S1: Schematic representation of the formation of an imine bond between GT-73 and Lys ${ }^{536}$ in PECAM-1, Figure S1: Mass spec determination of the covalent addition of a GT-73 fragment to PECAM-1.

Author Contributions: E.A., R.M., R.L., P.B., T.G., B.A.I. and A.G. designed the study. E.B., E.A., L.L., T.G. and S.Z. performed the experiments and the data analyses. A.G., B.A.I., E.B., E.A. and P.B. prepared the manuscript. T.G., E.A., P.B. and B.A.I. brought important intellectual content. All authors have read, reviewed, and approved the final submitted manuscript and agree to take public responsibility for the published results.

Funding: This research was funded by a Bar-Ilan University new faculty grant (to A.G.), Grant Number 182009.

Institutional Review Board Statement: Mice were handled according to the regulations formulated by the Israel Animal Care and Use Committee (Israel Ministry of Health). All experiments were approved by the Israeli Ministry of Health (the Animal Care Committee) and were conducted according to the Israeli National Veterinary Law.

Informed Consent Statement: Two healthy donors donated several milliliters of blood for the monocyte transmigration in vitro experiments.

Data Availability Statement: All supplemental data can be found at MDPI. In addition, we will share milligram amounts of GT-73 for biological tests. Any other data will also be made available to any investigator upon request.

Acknowledgments: We thank Steven Manch for the English editing and Nir London from the Weizmann Institute of Science, Rehovot, Israel, for his advice to use sodium borohydride to stabilize the covalent binding between GT-73 and PECAM-1. We also thank Marina Vainberg and Alexander Vainberg for providing the space and the scientific equipment/environment for this work.

Conflicts of Interest: The authors declare no conflict of interest.

Sample Availability: All supplemental data can be found at MDPI. In addition, we will share milligram amounts of GT-73 for biological tests. Any other data will also be made available to any investigator upon request. All other samples of the synthetized compounds also are available from the authors.

\section{References}

1. Qiu, H.; Tong, Z.; Ma, P.; Hu, M.; Peng, Z.; Wu, W.; Du, B. Intensive care during the coronavirus epidemic. Intensive Care Med. 2020, 46, 576-578. [CrossRef]

2. Stam, H.J.; Stucki, G.; Bickenbach, J. Covid-19 and post intensive care syndrome: A call for action. J. Rehabil. Med. 2020, 52, 19-22. [CrossRef]

3. Liew, M.F.; Siow, W.T.; MacLaren, G.; See, K.C. Preparing for CovID-19: Early experience from an intensive care unit in Singapore. Crit. Care 2020, 24, 83. [CrossRef] [PubMed]

4. Mehta, P.; McAuley, D.F.; Brown, M.; Sanchez, E.; Tattersall, R.S.; Manson, J.J. COVID-19: Consider cytokine storm syndromes and immunosuppression. Lancet 2020, 395, 1033-1034. [CrossRef]

5. Calcaterra, D.; Heather, B.; Kohl, L.P.; Erickson, H.L.; Prekker, M.E. Bedside veno-venous ECMO cannulation: A pertinent strategy during the COVID-19 pandemic. J. Card. Surg. 2020, 35, 1180-1185. [CrossRef] [PubMed]

6. Goh, K.J.; Choong, M.C.; Cheong, E.H.; Kalimuddin, S.; Duu Wen, S.; Phua, G.C.; Chan, K.S.; Haja Mohideen, S. Rapid progression to acute respiratory distress syndrome: Review of current understanding of critical illness from COVID-19 infection. Ann. Acad. Med. Singap. 2020, 49, 108-118. [CrossRef] [PubMed]

7. Li, X.; Ma, X. Acute respiratory failure in COVID-19: Is it "typical" ARDS? Crit. Care 2020, 24, 198. [CrossRef] [PubMed]

8. Zhang, J.W.; Hu, X.; Jin, P.F. Cytokine storm induced by SARS-CoV-2 and the drug therapy. Chin. Pharm. J. 2020, 55, 333-336. [CrossRef]

9. McKechnie, J.L.; Blish, C.A. The innate immune system: Fighting on the front lines or fanning the flames of COVID-19? Cell Host Microbe 2020, 27, 863-869. [CrossRef]

10. Getter, T.; Margalit, R.; Kahremany, S.; Levy, L.; Blum, E.; Khazanov, N.; Keshet-Levy, N.Y.; Tamir, T.Y.; Ben Major, M.; Lahav, R.; et al. Novel inhibitors of leukocyte transendothelial migration. Bioorg. Chem. 2019, 92, 103250. [CrossRef] 
11. Feng, Y.-M.; Chen, X.-H.; Zhang, X. Roles of PECAM-1 in cell function and disease progression. Eur. Rev. Med. Pharmacol. Sci. 2016, 20, 4082-4088.

12. Bergom, C.; Goel, R.; Paddock, C.; Gao, C.; Newman, D.K.; Matsuyama, S.; Newman, P.J. The cell-adhesion and signaling molecule PECAM-1 is a molecular mediator of resistance to genotoxic chemotherapy. Cancer Biol. Ther. 2006, 5, $1699-1707$. [CrossRef] [PubMed]

13. Goldberger, A.; Middleton, K.A.; Newman, P.J. Changes in expression of the cell adhesion molecule PECAM-1 (CD31) during differentiation of human leukemic cell lines. Tissue Antigens 1994, 44, 285-293. [CrossRef] [PubMed]

14. Nakada, M.T.; Amin, K.; Christofidou-Solomidou, M.; O’Brien, C.D.; Sun, J.; Gurubhagavatula, I.; Heavner, G.A.; Taylor, A.H.; Paddock, C.; Sun, Q.-H.; et al. Antibodies against the first Ig-like domain of human platelet endothelial cell adhesion molecule-1 (PECAM-1) that inhibit PECAM-1-dependent homophilic adhesion block in vivo neutrophil recruitment. J. Immunol. 2000, 164, 452-462. [CrossRef] [PubMed]

15. Bayat, B.; Werth, S.; Sachs, U.J.H.; Newman, D.K.; Newman, P.J.; Santoso, S. Neutrophil transmigration mediated by the neutrophil-specific antigen CD177 Is influenced by the endothelial S $536 \mathrm{~N}$ dimorphism of platelet endothelial cell adhesion molecule-1. J. Immunol. 2010, 184, 3889-3896. [CrossRef]

16. Muller, W.A. Transendothelial migration: Unifying principles from the endothelial perspective. Immunol. Rev. 2016, 273, 61-75. [CrossRef]

17. Chistiakov, D.A.; Orekhov, A.N.; Bobryshev, Y.V. Endothelial PECAM-1 and its function in vascular physiology and atherogenic pathology. Exp. Mol. Pathol. 2016, 100, 409-415. [CrossRef]

18. O'Brien, C.D.; Lim, P.; Sun, J.; Albelda, S.M. PECAM-1-dependent neutrophil transmigration is independent of monolayer PECAM-1 signaling or localization. Blood 2003, 101, 2816-2825. [CrossRef]

19. Yong, K.L.; Watts, M.; Shaun Thomas, N.; Sullivan, A.; Ings, S.; Linch, D.C. Transmigration of CD34+ cells across specialized and nonspecialized endothelium requires prior activation by growth factors and is mediated by PECAM-1 (CD31). Blood 1998, 91, 1196-1205. [CrossRef]

20. Shinde, V.N.; Dhiman, S.; Krishnan, R.; Kumar, D.; Kumar, A. Synthesis of imidazopyridine-fused indoles via one-pot sequential knoevenagel condensation and cross dehydrogenative coupling. Org. Biomol. Chem. 2018, 16, 6123-6132. [CrossRef]

21. Yang, X.; Yu, Y.; Xu, J.; Shu, H.; Xia, J.; Liu, H.; Wu, Y.; Zhang, L.; Yu, Z.; Fang, M.; et al. Clinical course and outcomes of critically ill patients with SARS-CoV-2 pneumonia in Wuhan, China: A single-centered, retrospective, observational study. Lancet Respir. Med. 2020, 8, 475-481. [CrossRef]

22. Meduri, G.U.; Annane, D.; Chrousos, G.P.; Marik, P.E.; Sinclair, S.E. Activation and regulation of systemic inflammation in ARDS: Rationale for prolonged glucocorticoid therapy. Chest 2009, 136, 1631-1643. [CrossRef]

23. Whyte, C.S.; Morrow, G.B.; Mitchell, J.L.; Chowdary, P.; Mutch, N.J. Fibrinolytic abnormalities in acute respiratory distress syndrome (ARDS) and versatility of thrombolytic drugs to treat COVID-19. J. Thromb. Haemost. 2020, 18, 1548-1555. [CrossRef]

24. Solinas, C.; Perra, L.; Aiello, M.; Migliori, E.; Petrosillo, N. A critical evaluation of glucocorticoids in the management of severe COVID-19. Cytokine Growth Factor Rev. 2020, 54, 8-23. [CrossRef]

25. Huang, X.; Xiu, H.; Zhang, S.; Zhang, G. The role of macrophages in the pathogenesis of ALI/ARDS. Mediat. Inflamm. 2018, 2018. [CrossRef] [PubMed]

26. Nasef, N.A.; Mehta, S.; Ferguson, L.R. Susceptibility to chronic inflammation: An update. Arch. Toxicol. 2017, 91, 1131-1141. [CrossRef]

27. Hausmann, J.S. Targeting cytokines to treat autoinflammatory diseases. Clin. Immunol. 2019, 206, 23-32. [CrossRef]

28. Zhang, J.-M.; An, J. Cytokines, inflammation and pain. Int. Anesth. Clin. 2009, 69, 482-489. [CrossRef] [PubMed]

29. Imanishi, J. Expression of cytokines in bacterial and viral infections and their biochemical aspects. J. Biochem. 2000, 127, 525-530. [CrossRef] [PubMed]

30. Song, P.; Li, W.; Xie, J.; Hou, Y.; You, C. Cytokine storm induced by SARS-CoV-2. Clin. Chim. Acta 2020, 509, 280-287. [CrossRef]

31. Polidoro, R.B.; Hagan, R.S.; de Santis Santiago, R.; Schmidt, N.W. Overview: Systemic inflammatory response derived from lung injury caused by SARS-CoV-2 infection explains severe outcomes in COVID-19. Front. Immunol. 2020, 11, 1626. [CrossRef] [PubMed]

32. Guo, Z.; Li, Q.; Han, Y.; Liang, Y.; Xu, Z.; Ren, T. Prevention of Lps-induced acute lung injury in mice by progranulin. Mediat. Inflamm. 2012, 2012, 540794. [CrossRef] [PubMed] 